\title{
Radio studies of gravitational lensing and future telescopes
}

\author{
Neal Jackson* \\ $J B C A$ /University of Manchester \\ E-mail: njj@jb.man.ac.uk
}

I review briefly the current status of studies of gravitational lensing, in particular current radio observations, and what lensing can tell us about mass distributions. VLBI observations of lenses to constrain central potentials of lensing galaxies and possible CDM substructure are presented, as well as ongoing efforts to measure the Hubble constant in radio lenses. Finally, I discuss efforts to use future telescopes, such as e-Merlin, to improve these results, and the possibilities of future surveys with telescopes such as LOFAR and the SKA.

From Planets to Dark Energy: the Modern Radio Universe October 1-5 2007

The University of Manchester, UK

* Speaker. 


\section{Introduction}

A gravitational lens is produced when a large foreground mass, such as a galaxy, lies close enough to the line of sight to some more distant galaxy or quasar. The light rays from the background object are bent by the foreground mass, and if the lines of sight coincide sufficiently accurately, multiple images of the background object are produced. Zwicky (1937) first suggested this as a consequence of Einstein's general theory of relativity, but the first example of gravitational lensing, the lensed quasar $0957+561$, was discovered by Walsh et al. (1979) as a result of a 966-MHz radio survey with the Lovell telescope.

Both systematic and serendipitous discoveries of lensed systems have been made since then, and the total number of reasonably secure lensed systems passed the 100 mark a few years ago; of the currently known lenses about a fifth have been discovered in radio surveys, and the remainder have come from optical observations. The most productive recent searches have been the SLACS project (Bolton et al. 2006) and the SDSS lensed quasar search (e.g. Inada et al. 2003), both of which have used the Sloan Digital Sky Survey as the starting point. Of the radio surveys, the largest was the CLASS survey, a collaboration between Jodrell Bank and institutes in the Netherlands and USA (Myers et al. 2003, Browne et al. 2003) which discovered 22 lens systems by observations of northern flat-spectrum radio sources.

Lens systems are important because they allow us to determine mass distributions in lensing galaxies independently of light output; they are therefore directly sensitive to dark matter at cosmologically interesting redshifts. In principle, one can use the images as constraints in an inverse problem to determine the overall mass structure of the lensing galaxy, to determine the degree to which the mass profile departs from smoothness, and to look at the central regions of the lensing galaxy. The problem in each case is that the lensing potential is only probed at the points in the lens plane where images are formed, and drawing astrophysical conclusions is usually non-trivial. A full review of the physics and phenomenology of lensing is given by Kochanek (2004), and the other main use of lensing - determination of the Hubble constant - is reviewed by Kochanek \& Schechter (2004) and Jackson (2007).

\section{Mass distributions: large-scale and in galaxy centres}

Of the lenses discovered by the CLASS survey, only a minority give useful constraints on the shape of the lensing galaxy's mass profile by themselves, for example in those cases with an Einstein ring (e.g. Wucknitz, Biggs \& Browne 2004) or multiple sources (e.g. Sykes et al. 1998, Cohn et al. 2001, Muñoz et al. 2001). This is because the lens galaxy's potential is only sampled at a few places. The SLACS survey, with its extended background galaxies, has provided many more well-constrained profiles recently (Koopmans et al. 2006). In nearly all cases, however, the overall mass profile is close to an isothermal distribution $\left(\rho \propto r^{-2}\right)$ within the range of a few kiloparsecs which is well-probed by lensing. This is roughly according to expectations, since the outer, dark-matter, slope should have a shallower profile (e.g. Navarro, Frenk \& White 1996) and the inner slope should be dominated by a steeper cusp where the baryons dominate the potential. The apparent universality of the isothermal profile is surprising, however. 


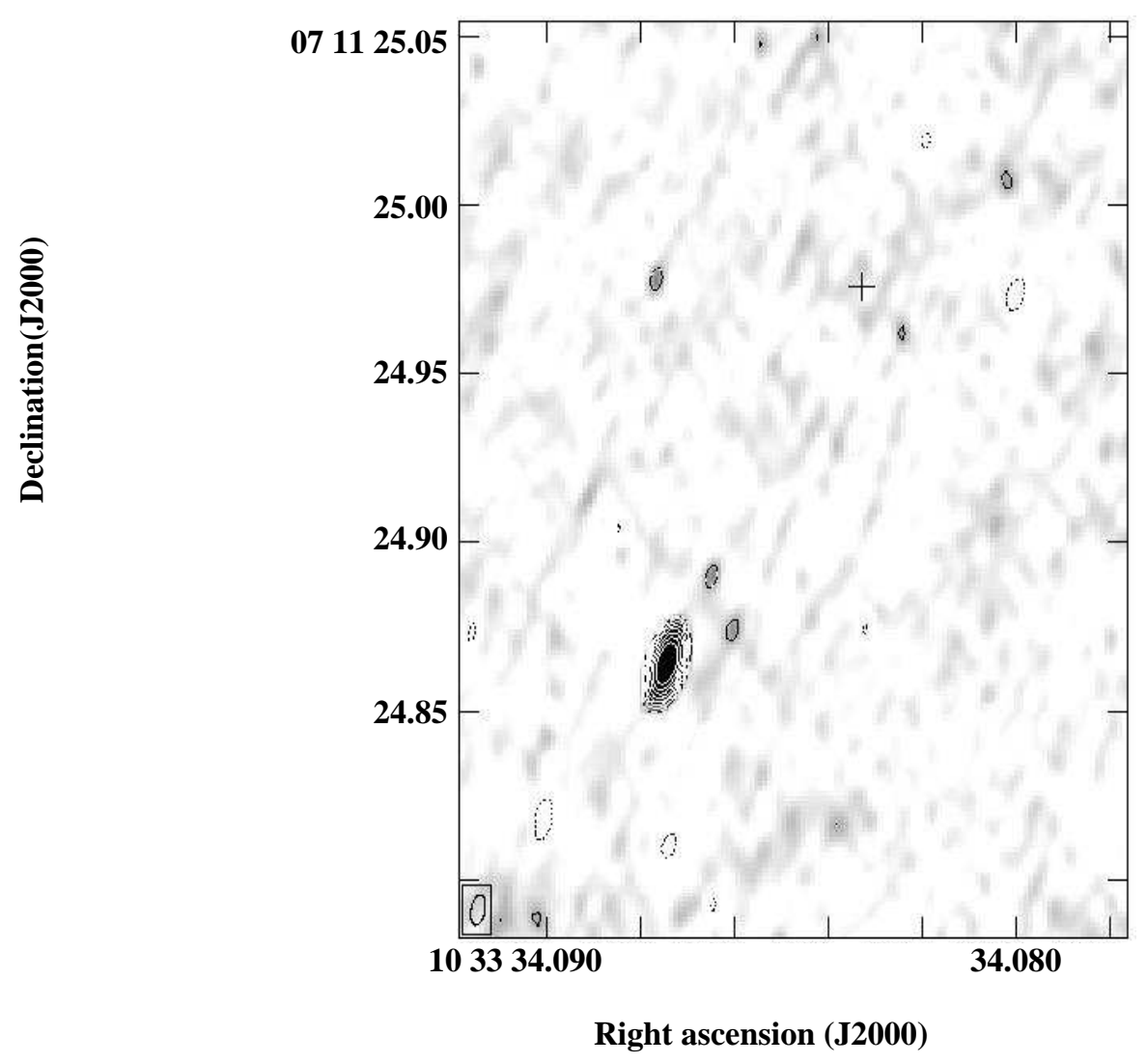

Figure 1: Observation of CLASS 1030+074 (Zhang et al. 2007) with the High Sensitivity Array (VLBA, VLA, Green Bank and Arecibo). The second image of the lens system is detected, but the central image (position indicated by a cross) is not.

The central $(<100 \mathrm{pc})$ region of galaxies is interesting because this is the region in which central star clusters and massive black holes dominate the potential, and whose properties correlate startlingly well with large-scale properties such as the bulge stellar velocity dispersion (Ferrarese $\&$ Merritt 2000), probably reflecting a profound truth about the way that galaxies form.

Many authors (e.g. Wallington \& Narayan 1993, Rusin \& Ma 2001) have pointed out that the absence or presence of a weak central image in a lensed system is a strong indicator of singularity, or lack of singularity, respectively, in the central potential. Using the only secure central image yet discovered, Winn, Rusin \& Kochanek (2003) constrain combinations of interesting parameters such as the inner galaxy power law slope and the break radius of any central cusp. In principle, more direct measurements of black hole masses and central cusp potentials are possible (Winn, Rusin \& Kochanek 2004, Mao, Witt \& Koopmans 2001, Keeton 2003) but would require detection of more than one central image, something not possible with current sensitivity. For example, Zhang et al. (2007) used a sensitive array of radio telescopes including the VLA, Green Bank and Arecibo and failed to detect the central image in CLASS 1030+074 (Fig. 1). 

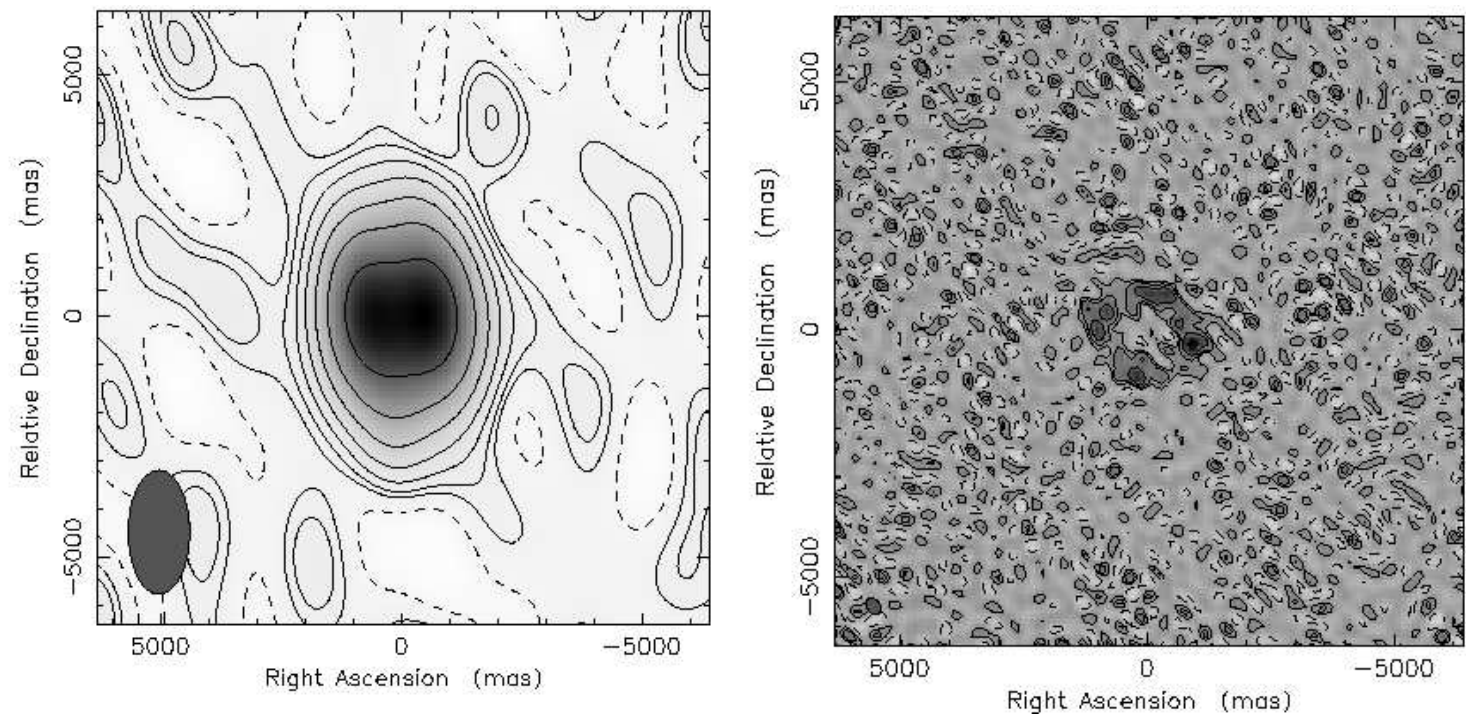

Figure 2: Simulation of a typical star-forming galaxy lensed by a foreground massive elliptical with a 0.8arcsecond Einstein radius, and imaged by the LOFAR instrument. Note that the core array (left) does not have the resolution to resolve this system; the full international array (right) is needed.

\section{The Hubble constant}

Refsdal (1964) pointed out that a lens system containing a variable source could provide a measurement of the Hubble constant. This is because measurement of a time delay between variations in brightness of the lensed image corresponds to a measurement of an absolute distance within the system. Given redshifts for the source and lens, this in principle gives a value for $H_{0}$, but does require that the lens mass model is known accurately, not an easy task given the paucity of observed constraints on many lenses. Currently, about 20 time delays are published; implications for $H_{0}$ are still a matter of debate (see e.g. Kochanek \& Schechter 2004 or Jackson 2007 for recent reviews).

\section{Future surveys}

There are two important developments which can be achieved by currently planned instruments.

First, study of existing lenses can be conducted with increased sensitivity. Since most lens systems have subarcsecond dimensions, the required resolution is of the order of 50-100 mas, especially for the detection of central images. The obvious instrument for doing this is e-MERLIN, since its resolution is well matched to the problem. The increase in sensitivity should allow routine detection of central images of about $0.01-0.1 \%$ of the flux density of the primary, sufficient to allow detection of central images in a significant fraction of known lenses (e.g. Keeton 2003). Increases in sensitivity in the upgrade of the VLA to the EVLA will also be important, as they will allow study of larger-scale properties of radio lens systems and the derivation of much better constraints on mass models.

Secondly, the statistical study of mass distributions in a large number of lens galaxies will require increasing the number of known lenses by factors of 10-100. Large optical surveys in view 
(such as the Large Synoptic Survey Telescope and the Gaia satellite) provide possible avenues for such large lensing programmes. In the radio, the most promising survey instrument in the near future is probably the Low Frequency Array (LOFAR), being built in the Netherlands; because of the long observing wavelength $(>2 \mathrm{~m})$ the arcsecond-resolution surveys to detect significant numbers of lenses will require baseline extensions to other European countries. The nominal sensitivity of this instrument will allow blind surveys over reasonable fractions of the sky down to noise levels of about 5-10 $\mu \mathrm{Jy} /$ beam, and should result in hundreds or thousands of lens systems. The much greater survey speed of the Square Kilometre Array, ten years further into the future, gives a corresponding increase in discovery speed.

\section{References}

[1] Bolton A.S., Burles S., Koopmans L.V.E., Treu T., Moustakas L.A. 2006, ApJ, 638, 703.

[2] Browne I.W.A., et al. 2003, MNRAS, 341, 13.

[3] Cohn J.D., Kochanek C.S., McLeod B.A., Keeton C.R. 2001, ApJ, 554, 1216.

[4] Ferrarese L., Merritt D. 2000, ApJ, 539, L9.

[5] Inada N., et al. 2003, Nature, 426, 810

[6] Jackson N. 2007, LRR, 10, 4.

[7] Keeton C.R. 2003, ApJ, 582, 17.

[8] Kochanek C.S., 2004, in Kochanek, Schneider \& Wambsganss, Proc. 33rd Saas-Fee Advanced Course, eds. Meylan et al (Springer)

[9] Kochanek C.S., Schechter P.L. 2004, Measuring and Modelling the Universe, Carnegie Obs. Cent. Symp., CUP, 117.

[10] Koopmans L.V.E., Treu T., Bolton A.S., Burles S., Moustakas L.A. 2006, ApJ, 649, 599.

[11] Mao S., Witt H.J., Koopmans L.V.E. 2001, MNRAS, 323, 301.

[12] Muñoz J.A., Kochanek C.S., Keeton C.R. 2001, ApJ, 558, 657.

[13] Myers S.T., et al., 2003, MNRAS, 341, 1.

[14] Navarro J.F., Frenk C.S., White S.D.M. 1996, ApJ, 462, 563.

[15] Refsdal S. 1964, MNRAS, 128, 307.

[16] Rusin D., Ma C.P. 2001, ApJ, 549, L33.

[17] Sykes C.M., et al., 1998, MNRAS, 301, 310.

[18] Wallington S., Narayan R. 1993, ApJ, 403, 517.

[19] Walsh D., Carswell R.F., Weymann R.J. 1979, Natur, 279, 381.

[20] Winn J.N., Rusin D., Kochanek C.S. 2003, ApJ, 587, 80.

[21] Winn J.N., Rusin D., Kochanek C.S. 2004, Natur, 427, 613.

[22] Wucknitz O., Biggs A.D., Browne I.W.A. 2004, MNRAS, 349, 14.

[23] Zhang M., Jackson N., Porcas R., Browne I.W.A., 2007, MNRAS, 377, 1623

[24] Zwicky, F., 1937, Phys Rev D, 51, 290 